\title{
Diagnostic et pronostic à l'époque d'Hippocrate et à la nôtre
}

\author{
Par Antoine Thivel
}

Pour nous modernes, même si nous ne sommes pas médecins, la distinction entre diagnostic et pronostic paraît parfaitement établie. Pour tout homme moyennement cultivé, il est évident que le diagnostic consiste à reconnaître une maladie chez un malade, à l'identifier, à la «déterminer», comme dit le dictionnaire Robert, tandis que le pronostic est le «jugement que porte un médecin, après le diagnostic, sur la durée, le déroulement et l'issue d'une maladie» (d'après le même dictionnaire). Et pour peu que nous ayons appris quelques mots de grec et de latin, nous savons que dans «diagnostic» il y a le préfixe «dia-» qui indique une séparation, et que par conséquent diagnostiquer une maladie, c'est essentiellement la distinguer, la séparer des autres, tandis que dans "pronostic» on trouve le préfixe «pro-» qui veut dire «avant», si bien que le pronostic est naturellement une prévision sur le cours ultérieur de la maladie, si possible sur son issue, heureuse ou malheureuse. Enfin, comme nous avons l'esprit scientifique, nous savons que dans l'exercice de la médecine le diagnostic est de loin l'acte le plus important, car il s'appuie sur toute la science médicale, anatomique, physiologique et pathologique, et il détermine le traitement; le pronostic, en revanche, est quelque chose de beaucoup plus aléatoire, soumis à un grand nombre de données inconnues ou impondérables, et il est rare que les médecins modernes se riquent à le prononcer avec assurance, surtout dans les cas graves.

Ces définitions, ces conceptions sont très répandues à notre époque, mais il est facile de constater qu'il n'en a pas toujours été ainsi.

Une première série de renseignements peut être tirée de l'histoire des mots eux-mêmes. Consultons le Dictionnaire étymologique de la langue française de O. Bloch et W.von Wartburg (P. U.F. 1968), nous trouvons à Pronostic:

«XIII ${ }^{\circ}$ (siècle), écrit alors pronostique, parfois féminin au $\mathrm{XV}^{\circ}$ s; pronostic depuis le $\mathrm{XVI}^{\circ}$. Emprunté de l'adjectif latin de basse époque prognosticus «de pronostic), issu de prognostica, pluriel neutre employé surtout comme titre d'ouvrage (du grec prognôstica, de même emploi, du verbe prognôstikein (connaître d'avance); de là aussi l'ancienne orthographe prognostic, conservée dans l'adjectif médical prognostique. Dérivés: pronostiquer, 1314; pronostiqueur, $\mathrm{XIV}^{\circ} . »$ 
Si le mot «pronostic» n'est pas attesté en français avant le $\mathrm{XII}^{\circ}$ siècle, c'est probablement parce qu'auparavant tous les ouvrages de médecine étaient écrits en latin, mais cela ne veut pas dire que la notion de pronostic était étrangère à la médecine de ce temps, au contraire! Les histoires de la médecine ${ }^{1}$ nous enseignent qu'au $\mathrm{XIII}^{0}$ et au XIV ${ }^{0}$ siècles la France, et l'Europe entière, vivaient sous le règne du galénisme; le pronostic était donc une partie essentielle de l'art médical, comme il l'a été dans toute l'antiquité, même si le diagnostic avait déjà commencé à faire, comme nous le verrons, de timides apparitions. Il est donc bon de savoir que le mot était couramment employé dès le $\mathrm{XIII}^{\circ}$ siècle, et que dès cette époque il commençait à avoir plusieurs dérivés.

Voici maintenant ce que le même dictionnaire étymologique nous dit à propos du diagnostic:

«Diagnostique, adj. 1584; écrit aussi -ic. Empr. du grec diagnostikos (apte à reconnaître). Dérivés: diagnostic, 1759, d'après la forme -ic de l'adj.; -tiquer 1836.»

Certes, l'histoire des mots ne reflète pas fidèlement l'histoire des idées, et l'on sait qu'un des dangers des études qui se fondent uniquement sur le vocabulaire est d'aboutir à des conclusions trop dogmatiques, quand leurs auteurs oublient qu'un même mot peut représenter plusieurs concepts et qu'inversement un même concept peut être exprimé par plusieurs mots; il faut aussi tenir compte de l'évolution du langage, qui fait que la relation entre les mots et les idées se modifie sans cesse. Mais ici, dans le cas du pronostic et du diagnostic, une chose est claire, c'est que la notion de diagnostic s'est formée beaucoup plus tardivement que celle de pronostic, puisqu'elle n'apparaît nettement qu'au milieu du $\mathrm{XVIII}^{\circ}$ siècle, et le verbe qui en est tiré au début du XIX ${ }^{0}$ siècle seulement, et lorsqu'on cherche des traces de l'adjectif correspondant, employé dans des expressions comme «signes diagnostiques» par opposition à «signes pronostiques», on ne les trouve qu'à la fin du XVI ${ }^{\circ}$ siècle.

Ce fait ne doit pas nous étonner si nous le replaçons dans l'ensemble de l'histoire de la médecine. Pour l'observateur objectif, il ne fait pas de doute qu'à la fin du Moyen-Age la médecine n'avait guère progressé depuis la fin de l'antiquité, plus particulièrement depuis l'époque de Galien: l'anatomie et la physiologie en étaient toujours au même point, elles s'appuyaient toujours sur la théorie des humeurs et des tempéraments, et la pathologie reposait sur des bases purement symptomatiques. On peut donc dire que les médecins de la fin du Moyen-Age n'identifiaient pas plus la maladie que ceux de 
l'antiquité, qu'ils n'étaient pas plus capables qu'eux de formuler de vrais diagnostics. Sans doute, l'observation clinique existait depuis longtemps, mais elle se limitait aux symptômes, elle ne se prononçait pas sur la nature de la maladie, qu'il n'était possible de cerner ni par une nosologie correcte, ni par une véritable notion de la spécificité étiologique. Pour pouvoir revenir aux bases de la clinique, et leur donner tout le soubassement expérimental nécessaire, il a fallu le travail de quatre siècles, du $\mathrm{XV}^{\circ}$ au $\mathrm{XVIII}^{0}$ siècle, pour qu'enfin au début du XIX ${ }^{0}$ siècle s'épanouisse la méthode anatomopathologique des Bichat, Laënnec et Magendie, qui a fait de la médecine une science, en attendant que la découverte de la bactériologie permette d'identifier et de combattre la cause d'un grand nombre de maladies.

Ecrire l'histoire du diagnostic, ce serait écrire l'histoire de toute la médecine. Un fait frappant, c'est que la médecine elle-même n'a pas pu être modifiée par les découvertes des anatomistes des $\mathrm{XV}^{\circ}$ et XVI $\mathrm{XV}^{\circ}$ siècles, ni par celles des physiologistes des $\mathrm{XVII}^{\circ}$ et $\mathrm{XVIII}^{\circ}$ siècles: jusqu'à Laënnec, Hippocrate et Galien sont restés les grandes autorités, Laënnec lui-même les a étudiés et critiqués, s'est appuyé sur eux pour faire ses propres découvertes, et lorsque Littré, en 1839, a commencé sa grande édition d'Hippocrate, il se disait que son travail serait avant tout utile aux médecins; chemin faisant, en éditant les textes, il discute de la justesse d'une prescription de saignée ou de lavement, ou de tel ou tel diagnostic, qui nous paraît aujourd'hui complètement dépassé; visiblement, pour lui, malgré tout l'apport de l'anatomie et de la physiologie des siècles classiques, la médecine était toujours la même qu'au $\mathrm{V}^{\mathbf{0}}$ siècle avant J. C. en Grèce; il ne se rendait absolument pas compte que, pendant qu'il faisait son travail d'édition, des découvertes capitales, qui allaient bouleverser complètement la médecine, étaient en train de se produire.

Ce retard de la pathologie et de la clinique, c'est-à-dire de la médecine à proprement parler, sur les sciences qui nous ont fait connaître l'organisme humain, ainsi que sur la chimie et la biologie, ne doit pas être uniquement imputé à la paresse de l'esprit humain et au poids des institutions. Certes, la résistance de la médecine traditionnelle a été très forte (on se rappelle avec quelle mauvaise foi Guy Patin, au temps de Molière, prétendait réfuter Harvey, affirmant fièrement et en latin qu'il préferait se tromper avec Galien plutôt que de croire à la circulation avec le médecin anglais), mais les connaissances anatomiques et physiologiques n'avaient que peu d'incidences sur le diagnostic et le traitement des maladies. Pour que les deux soient réunies, la méthode clinique selon Hippocrate et la connaissance du 
corps humain et de ses affections, il a fallu l'étude patiente sur les cadavres des lésions provoquées par les maladies, étude dont déjà Galien, au II $^{\circ}$ siècle de notre ère, avait une idée, puis qui a progressé au $\mathrm{XVII}^{\circ}$ siècle avec Sydenham et Baglivi, et au XVIII' ${ }^{\circ}$ siècle avec G. B. Morgagni. Ainsi la liaison s'est faite peu à peu entre l'ancienne méthode d'observation des malades, qui était mise au point depuis l'antiquité, mais qui ne reposait pas sur des bases positives, et les connaissances nouvelles accumulées par les chercheurs depuis quatre siècles, et qui enfin trouvaient leur justification. Ce qui s'est formé au cours de cette période, et qui a permis à la médecine de faire ce saut qualitatif vers l'avant, c'est la notion de spécificité: désormais la maladie n'est plus un ensemble de symptômes plus ou moins contingents, liés à la saison, à la situation du malade, à son tempérament, et à diverses causes occasionnelles, c'est un état créé par un agent pathogène bien déterminé, qui se définit comme une entité unique, et se combat par des moyens spécifiques. A partir de là peuvent être jetés les fondements d'une véritable nosologie, ce qui n'est pas un défaut de la médecine ancienne, mais toujours un des buts de la médecine expérimentale.

Sans doute, lorsque Laënnec reconnaît qu'il n'y a pas trente-deux phtisies, comme le disaient certains médecins de son temps, mais une seule, il fait faire un grand progrès à la pathologie en identifiant la maladie qu'il n'appelle pas encore la tuberculose, mais il ne connaît pas l'agent pathogène spécifique, qui sera découvert peu de temps après par Koch, et il n'a pas non plus le moyen de le combattre par la découverte ultérieure de Fleming, la pénicilline. Mais il n'en reste pas moins que toutes ces découvertes se tiennent, se suivent dans le temps et logiquement, chaque nouvelle voie ouverte rendant nécessaire d'en ouvrir une autre. On entend dire quelquefois - d'une manière certainement abusive - que de nos jours la médecine change tous les dix ans (ou plus rapidement encore), que les médecins, pères d'étudiants en médecine, ne reconnaissent pas la médecine qu'on enseigne à leurs enfants. Sans doute, dans ce domaine comme dans les autres, le progrès s'accélère, les moyens d'investigation et la thérapeutique se renouvellent à une vitesse vertigineuse, mais l'impulsion de la médecine moderne, qui contenait en germe toutes ses mutations ultérieures, a été donnée au début du $\mathrm{XIX}^{0}$ siècle par l'école des anatomo-pathologistes, qui a profité des travaux antérieurs, mais en a fait une synthèse décisive. Ce n'est pas parce que le diagnostic de Laënnec est incomplet (il ne connaît pas les bactéries) qu'il faut le taxer d'archaïsme: désormais il a surmonté les difficultés qui faisaient obstacle au diagnostic dans les époques précédentes, et il instaure 
une méthode qui ne tardera pas à porter ses fruits. Quant aux maladies endogènes, celles qui ne sont pas provoquées par un agent extérieur mais par un déséquilibre des fonctions, il ne faut pas dire qu'elles mettent en cause la spécificité, mais que leur spécificité est d'un ordre différent de celle qui a fait la gloire de la médecine du $\mathrm{XIX}^{0}$ siècle: c'est le domaine encore largement inconnu des sécrétions des glandes endocrines, et des rapports de l'individu avec son milieu, les deux étant d'ailleurs - selon toute probabilité étroitement liés. Dans ces maladies, le diagnostic est plus difficile, mais cela ne veut pas dire qu'on doive renoncer à l'établir.

Telle est, très rapidement rappelée, la situation du diagnostic dans la médecine moderne, à la suite de toutes les découvertes qui ont fait de la médecine une science depuis environ deux siècles. L'histoire des mots «diagnostic» et "pronostic» le montre: le diagnostic, c'est-à-dire la description de la maladie en tant que telle et la recherche de sa cause, ne s'est dégagé que très lentement et très difficilement de sa nébuleuse primitive, l'acte médical complexe et passablement confus, fait d'intuition aussi bien que de rapprochements rationnels, qu'on appelait en médecine ancienne le «pronostic». On sait que cette évolution n'a été rendue possible que par les progrès de l'anatomie et de la physiologie, de la physique, de la chimie et de la biologie, par la constitution de la science expérimentale reposant sur le déterminisme, quand on se fût avisé que les phénomènes de la vie obéissaient à des lois aussi strictes que ceux de la matière inerte. C'est là la conviction de savants comme Claude Bernard, qui redoutait avant tout le retour de la moindre trace de vitalisme, ce qui lui fit dans un premier temps refuser la découverte de Pasteur, parce qu'il croyait que ces petits êtres vivants, les microbes, devaient quelque chose à l'imagination de leur inventeur. Sans doute, le déterminisme appliqué aux phénomènes de la vie n'est pas le dernier mot de la science, et à notre époque il ne manque pas de chercheurs et de théoriciens pour essayer de remettre en cause cette doctrine, surtout depuis que les théories de la relativité ont prouvé, en physique, leur vérité et leur efficacité. Mais est-ce une raison pour revenir à la médecine d'avant Laënnec, pour prôner l'art du pronostic, la connaissance intime de chaque cas particulier en dehors de la connaissance des causes, comme au temps d'Hippocrate? C'est un fait que même ceux qui, dans le public, critiquent la médecine dite «officielle» et, sous l'influence de charlatans ou de faux savants, veulent revenir à la médecine ancienne, voire à la médecine primitive et à la magie, sous prétexte que c'est plus «naturel», font appel à la médecine scientifique lorsqu'ils sont atteints d'une maladie grave et craignent pour leur vie. Ainsi, 
le problème historique des rapports entre le pronostic et le diagnostic se double d'un problème de fond: si nous avons deux médecines, l'une qui cherche les causes des maladies et tend à les détruire par une intervention au besoin violente, l'autre qui se préoccupe avant tout de l'état général du malade et se propose seulement d'aider la nature dans son travail de rétablissement de la santé, laquelle est dans le vrai, à laquelle pouvons-nous faire confiance? Nous croyons que l'histoire du concept de pronostic peut nous aider à nous faire une opinion dans ce débat, aussi ancien que la médecine elle-même.

A notre époque, le pronostic est un acte médical conforme à l'étymologie du mot: c'est la prévision de l'évolution de la maladie et de son issue. Mais nous allons voir que curieusement, dans la médecine ancienne, là où le pronostic avait le plus d'importance, il différait grandement du nom qu'on lui avait attribué et de son sens étymologique. Pour comprendre cela, il faut étudier l'histoire du mot en latin et en grec, et l'on s'aperçoit que, bien que le vocabulaire ne soit pas fixé, la notion de pronostic dans la médecine ancienne remonte, comme on pouvait s'y attendre, à la définition qu'en ont donnée Hippocrate et ses disciples dans le traité du Pronostic et dans les Epidémies.

La formule classique se trouve dans Epid. I, 5, seconde constitution de

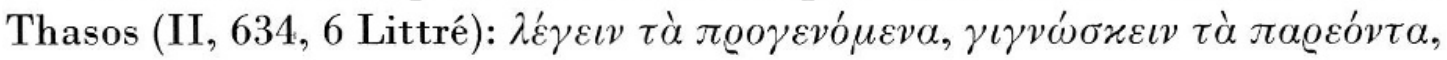

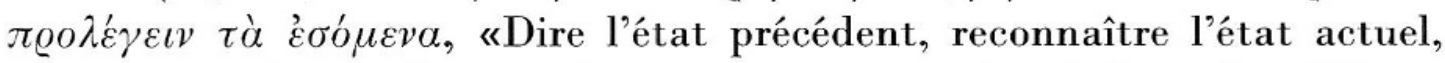
prédire l'état futur». La suite du texte contient nombre de formules célèbres: «Prendre garde à deux choses dans les maladies, être utile ou du moins ne pas nuire. L'art consiste en trois termes, la maladie, le malade, le médecin; le médecin est le serviteur de l'art: il faut que le malade s'oppose à la maladie avec l'aide du médecin.»

Cette définition du pronostic s'accorde en tous points avec celle que donne le traité du Pronostic en son premier paragraphe (II, 110, 2-4 Littré):

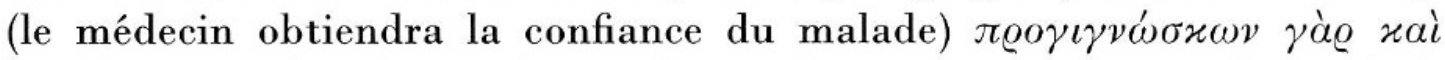

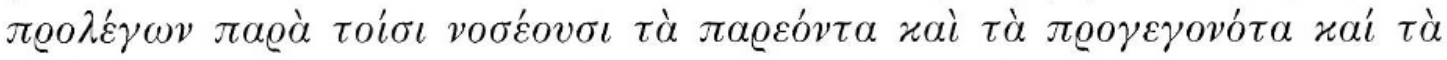
$\mu \dot{\varepsilon}^{\prime} \lambda \lambda \sigma \nu \tau \alpha$ '́ $\sigma \varepsilon \sigma \theta \alpha$, «en reconnaissant d'un seul coup d'œil et en prédisant aux malades leur état présent, leur état passé et leur état futur». Le pronostic ne consiste donc pas seulement à prévoir, à prédire l'état futur du malade, il englobe le passé, le présent et l'avenir, si bien qu'on a scrupule à traduire $\pi \varrho o \gamma \iota \gamma \nu \omega \sigma \varkappa \omega$ et $\pi \varrho o \lambda \varepsilon \dot{\gamma} \omega$ par «prévoir» et «prédire», comme si le préfixe $\pi \varrho o$ - n'avait pas la même valeur dans ces mots employés par Hippocrate que dans la signification moderne du mot «pronostic». Dans le dernier cas, nous avons traduit $\pi \varrho o \gamma \iota \gamma \nu \omega ́ \sigma \varkappa \omega$ par «reconnaître d'un seul coup d'œil», et c'est 
bien de cela qu'il s'agit, plutôt que de prévisions sur la guérison ou la mort. Le médecin doit être capable de reconnaître d'avance, dès l'abord, au préalable toute la nature de la maladie, se prononcer du premier coup, dès le premier examen, embrasser dans son ensemble l'état du malade, grâce aux règles qui sont consignées pour lui dans le traité. Et l'on sait que le pronostic hippocratique est très vaste: le médecin doit reconstituer toute la maladie dans l'ensemble de son histoire, et pour cela examiner le visage, la position du corps, les gestes des mains, les sueurs et l'état de la peau, la nature des selles et des urines, les vomissements et les crachats, les conditions spéciales de temps et de lieu, la nature de la saison par rapport à ce qu'elle devrait être, la qualité des aliments, des eaux... etc. Comme dit Littré dans son introduction au Pronostic (II, 108): le pronostic hippocratique se rapproche moins de notre moderne diagnostic, basé sur une exacte sémiologie, que de ce qu'on appelle «le flair», «le sens médical». Ailleurs, il dit aussi que cette considération de l'état général du malade et de la marche que doit suivre logiquement la maladie, étant donné l'état très rudimentaire où se trouvaient l'anatomie et la physiologie, était «le seul moyen d'éviter de se perdre dans le dédale des faits particuliers et des conjectures, d'éviter la routine et l'empirisme; la prognose est la première construction scientifique que nous connaissions de la médecine» (I, 455 L.).

Des affirmations péremptoires de ce genre sont toujours dangereuses, et le moins qu'on puisse dire, c'est que cette appréciation de Littré est ambiguë, car d'une part il excuse Hippocrate de s'être servi de la méthode imparfaite du pronostic eu égard à l'état peu avancé de la science de son temps, et d'autre part il fait l'éloge du pronostic hippocratique comme étant la première forme scientifique de la médecine. En dehors du fait qu'on pourrait lui objecter que les médecines qui ont précédé la médecine grecque, celles de Mésopotamie et d'Egypte, avaient déjà de nombreux caractères positifs et même scientifiques, ce dernier adjectif pose un problème: que faut-il entendre par «scientifique»? On peut comprendre le mot au sens large ou au sens étroit; aus sens large, sont scientifiques tous les efforts que l'homme a faits depuis les origines pour essayer de comprendre le monde et d'agir sur lui, et à ce titre on pourrait citer la médecine primitive, avec son mélange de magie et de connaissances réelles, comme une première tentative de médecine scientifique, comme la science de cette époque; au sens étroit, le mot «scientifique» ne peut désigner que la science expérimentale, et en médecine il ne peut s'appliquer qu'à la médecine européenne à partir du $\mathrm{XIX}^{\circ}$ siècle. 
Littré, sans doute, n'allait pas jusqu'à attribuer au pronostic hippocratique la valeur de la médecine expérimentale, mais plus récemment nous avons vu cette opinion affirmée dans un ouvrage célèbre, qui reste une des meilleures introductions à Hippocrate, Observation et expérience chez les médecins de la Collection hippocratique, de Louis Bourgey (Paris, Vrin, 1953). Distinguant parmi les traités de cette Collection ceux qui se lancent dans des spéculations aventureuses' (les traités des Vents, des Chairs, des Semaines, du Régime) et d'un autre côté ceux qui pèchent par empirisme et ne présentent que des accumulations de faits sans aucune réflexion ou sans réflexion valable (Maladies II, Maladies III, Affections internes, les traités qu'on appelle «cnidiens» et ceux qui leur sont apparentés), l'auteur en vient ensuite à l'analyse des traités qui lui paraissent animés par une véritable pensée médicale, c'est-à-dire par l'union de la pensée et de l'expérience, et qui, pense-t-il, ont dû être rédigés par le groupe des médecins de Cos sous la direction d'Hippocrate, le plus génial d'entre eux. L'analyse du pronostic hippocratique est au centre de cette étude, et L.Bourgey, tout en lui reconnaissant certraines imperfections (la négligence des causes, par exemple), n'a pas d'expressions assez élogieuses pour cette attitude d'esprit. Le vaste programme que représente le pronostic ainsi conçu lui paraît une preuve de largeur de vues en même temps que de rigueur, d'humilité devant les faits; tout est pris en compte, les moindres détails comme l'ensemble, et le médecin hippocratique arrive à une compréhension intime de l'être vivant, considéré à la fois comme un tout et dans sa complexité. Nous lisons, p. 222:

\footnotetext{
«En lisant le Pronostic, et davantage encore les Epidémies I et III (car dans ce dernier ouvrage on saisit directement le praticien dans l'exercice de son art), on se rend compte que presque à chaque fois, en face de la maladie ondoyante et diverse, un effort nouveau d'intelligence est demandé. Cet effort, qui tend à reconstituer d'une manière non pas abstraite et générale, mais concrète et vivante l'histoire complète de la maladie, revêt une très grande portée; par lui le médecin devient capable d'une vision d'ensemble, d'une idée particulière fondée sur les faits.»
}

Cette idée «concrète et particulière» qui n'est pas «abstraite et générale» caractérise peut-être assez bien le pronostic hippocratique, mais il faut reconnaître qu'elle est plus près du «flair» et du «sens médical» dont parlait Littré (nous dirions aujourd'hui de la «mètis» après la belle étude de J.P. Vernant) que de la véritable connaissance positive et scientifique. Quelles que soient la bonne volonté d'un médecin et son attention en observant le malade, il ne peut inventer tout seul une pathologie qui demandera des siècles pour être mise au point, et il ne peut pas non plus la trouver par hasard. 
Mais la véritable pensée de L. Bourgey apparaît à la fin de son ouvrage, quand il s'efforce de définir la «médecine rationnelle» par opposition à la médecine théorique et à la médecine empirique. Voici ce qu'il écrit, p.261:

\begin{abstract}
«Une distinction devenue familière à certains penseurs contemporains, celle d'expliquer et de comprendre, peut, transportée dans le domaine de la vie, aider à mieux saisir l'attitude des médecins de Cos... (suit une critique du souci d'explication et de la recherche des causes)

L'attitude de compréhension, laissant de côté tout recours à des conceptions théoriques générales, essayerait de pénétrer l'intimité de l'être vivant en s'aidant de celui-ci seulement, en saisissant par exemple à la suite d'une réflexion prolongée que chaque partie du corps est liée aux autres et les soutient; une telle connaissance peut sans doute n'être qu'une affirmation banale suggérée par la première observation venue, mais elle peut exprimer aussi un savoir pensé et vécu qui détermine toute un manière d'agir et qui est plus riche et plus profond que sa traduction par les mots courants.»
\end{abstract}

Une telle conception n'a plus grand'chose à voir avec la médecine, et même avec la science. Nous ne pensons pas que les diagnostics des maladies infectieuses et les procédés d'anesthésie, d'asepsie, de lutte contre l'infection aient pu être trouvés par un simple regard intuitif, si prolongé fût-il. Qu'on le veuille ou non, la médecine est bien obligée d'avoir recours à la raison discursive, et cela était vrai à l'époque d'Hippocrate comme à la nôtre. Si l'intuition joue en effet un rôle dans l'acte médical, elle ne peut s'exercer avec quelques chances de sûreté que sur la base de toutes les connaissances précises acquises par les générations antérieures et par le praticien lui-même, elle ne saurait en aucun cas les remplacer, pas plus que la «compréhension» ne peut jouer le rôle d'une véritable explication.

Dans toute cette démonstration, il semble que L. Bourgey, comme il veut à tout prix réhabiliter le pronostic hippocratique et le présenter comme une préfiguration de la médecine scientifique moderne, compte pour mérites à Hippocrate des éléments qui sont en réalité les traits les plus négatifs de cette ancienne forme de médecine. Ainsi, il loue les médecins de Cos d'avoir une vue synthétique où tout entre en ligne de compte: la saison, le lieu, les habitudes du malade, la position de son corps, la couleur des urines, des selles... etc. Mais c'est justement cet immense inventaire qui nous paraît manquer de rigueur et rendre toute synthèse impossible, et qui plus est, dans cette pratique du pronostic, tout est mis sur le même plan: le fait que le malade arrache les brins de sa couverture est sans doute le signe d'une grande agitation, donc d'une maladie grave, mais il ne contient pas autant de précision que l'examen des urines. Hippocrate, en somme, met sur le même 
plan les observations générales et les symptômes particuliers. Il n'a pas le sens de la spécificité de la maladie. Nous ne disons pas cela pour diminuer ses mérites, mais pour essayer de voir quelle place il occupe exactement dans l'histoire de la médecine.

Enfin, L. Bourgey ne peut s'empêcher d'examiner (op.cit.pp.238-251) la coction, les crises, les jours critiques, les dépôts, les récidives, les paroxysmes, et la théorie des humeurs, tous concepts qui faisaient la base de la physiologie et de la pathologie à cette époque, et qu'Hippocrate, comme tous les médecins de son temps, a admis ${ }^{2}$. Mais chaque fois notre auteur s'efforce de démonter que lorsque ces concepts sont employés par Hippocrate et son école, ce ne sont pas vraiment des théories, mais une manière de saisir les faits, si bien que la médecine moderne pourrait encore avoir in térêt à utiliser ces notions. C'est ainsi qu'il déclare (p.249) que la théorie des humeurs a peut-être des imperfections, mais qu'elle montre que ces médecins avaient compris «l'importance pour la santé des masses liquides de l'organisme». On se demande ce que la médecine moderne pourrait tirer d'une idée aussi vague, et il faut d'ailleurs ajouter que dans la médecine ancienne les humeurs jouaient un grand rôle, et les discussions à ce sujet étaient nombreuses et passionnées. C'était ce que nous appelons un système empirique: quand on ne connaît pas la cause d'un phénomène, on en imagine une; les Grecs ont inventé la bile et le phlegme comme les Egyptiens ont imaginé des souffles, et les Chinois des zones dans le corps humain. Toutes ces notions n'ont aucune réalité anatomique ni physiologique, ce sont simplement des instruments intellectuels que les savants de ce temps se sont forgés pour pouvoir faire de la médecine en suppléant à leur manque d'information. Imaginer des humeurs à la place des anciens démons, c'est déjà un progrès, mais ce n'est pas encore la cause réelle. Il en est de même des notions de crise, de coction et de jours critiques: la médecine grecque est en progrès sur ce point par rapport aux médecines qui l'ont précédée, celles de Mésopotamie et d'Egypte, parce qu'elle considère la maladie comme un processus, et il est probable qu'elle a tiré cette idée de la physique ionienne, qui avait fait notablement progresser les connaissances en analysant toutes sortes de phénomènes astronomiques et météorologiques comme des processus, des séries de causes et d'effets gouvernées par le principe des semblables ou le principe des contraires. Mais il est évident qu'on essayait de fixer la crise en comptant les jours (procédé qui sera plus tard refusé par la médecine romaine, mais reviendra en honneur au Moyen-Age) pour la bonne raison qu'on ne connaissait pas la maladie, et encore moins sa cause. Littré a eu 
raison de souligner que cette idée d'une périodicité des fièvres (qui est présente aussi en Inde) a dû être suggérée par ce qu'il appelle «les fièvres rémittentes et semi-continues des pays chauds», c'est-à-dire les diverses formes de paludisme. Mais toutes les maladies aiguës ne sont pas périodiques, et pourtant on cherchait partout la périodicité, car cela correspondait à certaines idées cosmologiques, et il est assez comique de penser qu'à l'époque de Molière, des médecins exerçant en France et se réclamant d'Hippocrate continuaient à calculer les crises et les jours critiques alors que bien peu de leurs malades, sans doute, devaient être atteints de fièvres palustres.

Quant à l'idée de la coction, elle est également d'origine cosmologique: c'est le résultat d'une vaste analogie entre l'univers et l'homme, où les humeurs à l'intérieur du corps sont assimilées aux nuages et aux pluies, obéissent aux mêmes lois de condensation et de raréfaction. L'équilibre des humeurs, c'est la santé; celui de masses d'air, c'est le beau temps. Inversement, le dérangement du système donne la maladie dans un cas, l'orage dans l'autre. Ces idées sont sous-jacentes à toutes les conceptions des médecins du temps d'Hippocrate, et le maître de Cos lui-même n'y a pas échappé. Il est vain de chercher à distinguer les écrits en montrant ceux où la théorie tient moins de place et ceux où elle en tient plus, voire des traités qui ne contiendraient que des faits accumulés sans ordre, sans aucune théorie. Le propre de l'empirisme, nous le savons, c'est toujours de superposer à la collecte des faits des échafaudages de systèmes complètement arbitraires, et en l'occurrence, dans la médecine ancienne, des analogies illusoires entre l'homme et l'univers: comme on ne comprend pas ce qui se passe à l'intérieur du corps, on cherche à l'expliquer par la comparaison avec le monde extérieur. Il faut donc nous rendre à l'évidence: le pronostic hippocratique n'est pas une préfiguration de la médecine moderne, c'est une synthèse de toutes les observations cliniques qu'a faites Hippocrate, aidé de ses fils et de ses élèves (peut-être faut-il voir dans cette mise au point de l'observation clinique le principal apport qui a fait la célébrité du maître de Cos, et cet apport est inestimable), mais dans la mesure où il repose sur un système empirique, le pronostic prend place, avec la coction, les crises et les jours critiques, parmi les théories qui ont remplacé longtemps la science véritable et qui l'ont empêchée de se former.

D'ailleurs, pour être juste, il faut renoncer aux idées trop schématiques, et admettre que le pronostic hippocratique n'est pas étranger à la notion de maladie et à la recherche des causes. Nous avons déjà vu, dans un passage célèbre d'Epidémies $I$, que la médecine se composait de trois termes, le 
malade, la maladie, le médecin ${ }^{3}$. Donc, même dans un traité réputé «coaque», un traité qui est peut-être d'Hippocrate, la maladie n'est pas ignorée, elle est traitée sur un pied d'égalité avec le malade. On retrouverait cette idée dans de nombreux autres passages, si bien qu'il apparaît tout à fait artificiel d'opposer la «médecine de Cos», qui reposerait uniquement sur le pronostic et se désintéresserait de la nature de la maladie, et la «médecine de Cnide» qui chercherait seulement à identifier des maladies sans se soucier du malade. Nous retrouvons ici la difficile question des écoles de médecine dans la Collection hippocratique, et en particulier la tentative qui a été faite depuis longtemps, à partir du premier paragraphe du Régime dans les maladies aiguës, de reconstituer une «école de Cnide» dont les doctrines auraient été opposées en tous points à celles d'Hippocrate. Il est facile de voir que Littré, qui a fortement contribué à accréditer cette théorie, a cédé à une illusion historique: il a vu dans la polémique du premier paragraphe de $R$. $M$. $A$. le même débat qui opposait en son temps les créateurs de l'anatomo-pathologie comme Laënnec, et ceux qui refusaient les nouvelles découvertes en invoquant Hippocrate et en stigmatisant les chercheurs par cette formule: «Vous ne soignez que des maladies, nous soignons des malades.» Evidemment, c'est bien plus beau! Mais il est heureux que cette formule hypocrite n'ait pas triomphé, car si elle l'avait fait on ne compterait pas les millions de malades qui auraient continué à mourir d'appendicite, de rage ou de tuberculose. Il est évident qu'un débat entre «Coaques» et «Cnidiens» n'a pas pu avoir lieu au $\mathrm{V}^{0}$ siècle avant J. C., puisqu'à cette époque, comme nous sommes en train de le démontrer, la distinction entre diagnostic et pronostic n'existait pas. Dira-t-on alors que les «Coaques» croyaient plus à la médecine d'expectation et que les «Cnidiens» étaient plus interventionnistes? C'était aussi une idée de Littré, mais l'examen attentif des textes prouve qu'il n'en est rien, que dans les traités supposés «coaques» les incisions et cautérisations sont aussi nombreuses et douloureuses que dans les traités considérés comme «cnidiens», et inversement on trouve les mêmes principes d'expectation dans les uns que dans les autres (crises, jours critiques). Tout cela est normal, aucun médecin, aucune école de médecine ne pouvait à cette époque anticiper sur la médecine de l'avenir. Il se révèle d'ailleurs que Littré n'a pas été le premier à faire cette hypothèse sur l'opposition entre les écoles de Cnide et de Cos, et qu'à chaque époque les historiens de la médecine ont imaginé ce débat à l'image des débats qui agitaient les médecins de leur temps: un chercheur anglais, I. M. Lonie, qui avait commencé par concacrer des études très précises au problème de Cnide et Cos dans la Collection hippocratique, a 
montré ensuite que cette idée était due en réalité aux historiens de la médecine, d'après la conception qu'ils se faisaient de la science ${ }^{4}$; pour Daniel Le Clerc (Genève, 1696), Hippocrate a créé la médecine parce qu'il a su allier «la raison des philosophes» à l'expérience des Asclépiades, tandis que les Cnidiens étaient des empiristes (et cette idée remonte beaucoup plus haut, puisqu'on la trouve déjà au $\mathrm{XIV}^{\mathrm{o}}$ siècle chez le médecin italien Bertuccio, le maître de Guy de Chauliac); pour J. H. Schulze (Leipzig, 1728), les Cnidiens ont une conception théorique et générale de la thérapeutique, tandis qu'Hippocrate sait mieux adapter le traitement aux cas individuels; au $\mathrm{XVIII}^{\circ}$ siècle Haller et Grüner estiment que les Cnidiens n’ont pas su diviser les maladies et n'ont pas vu la maladie comme un processus; ce sera aussi l'opinion de K. Sprengel (Leipzig 1789-92), qui voit les Cnidiens comme des empiristes et de mauvais nosologistes, qui ont ignoré le pronostic, la doctrine des crises et de la coction, «la partie la plus admirable de la médecine hippocratique», dit-il. I. M. Lonie conclut en disant qu'à chaque époque les Cnidiens ont servi de faire-valoir à Hippocrate, le médecin idéal qui n'est pas tombé dans les défauts de l'empirisme, mais a su allier l'expérience à la théorie. Tout cela, évidemment, vient de la conception aristotélicienne de la science, mais ne contient aucun noyau historique ${ }^{5}$. Il faut ramener à ses justes proportions la polémique du premier paragraphe du Régime dans les maladies aiguës: l'auteur (qu'il soit Hippocrate ou un autre) ne reproche pas aux auteurs des Sentences cnidiennes d'avoir une nosologie (il en a une luimême), mais d'avoir une nosologie trop compliquée, de trop subdiviser les maladies, et en même temps d'avoir une thérapeutique trop simple. Il est donc partisan d'une nosologie plus simple et d'une thérapeutique plus différenciée ${ }^{6}$. Cette discussion prend place dans le cadre du pronostic hippocratique, que pratiquent aussi bien l'auteur de R.M.A. que ses adversaires ?

Une fois éliminés les faux problèmes qui ont longtemps occupé la critique hippocratique autour de la question de «Cnide et Cos?», il faut reconnaître que dans toutes les parties de la Collection hippocratiques, aussi bien dans les traités qui doivent être les plus proches d'Hippocrate et de ses disciples que dans ceux qui doivent être les plus éloignés, la recherche des causes tient une grande place, ainsi que les tentatives pour identifier les maladies. On pourrait citer de nombreux passages tirés de l'Ancienne médecine, d'Airs Eaux Lieux, d'Epidémies II, de l'Art, d'Affections, des Lieux dans l'homme, de Nature de l'homme, des Maladies des femmes, qui sont de véritables définitions du diagnostic ${ }^{8}$, et il ne faut pas nier que, même dans les 
descriptions de maladies qui prétendent s'en tenir à l'observation des symptômes, des diagnostics sont souvent tentés. Ainsi, dans les Epidémies, le paragraphe qui décrit le cas clinique est souvent terminé par le nom de la maladie, ou par des caractères conventionnels qui indiquent une hypothèse: le malade a dû guérir parce que les selles, les urines, les sueurs ont été abondantes, ou au contraire il est mort par suppression des selles ou des urines. Si l'on met ensemble, dans les Epidémies $I$ et $I I I$, les descriptions de cas particuliers et les «constitutions» ( $\alpha \alpha \tau \alpha \sigma \tau \alpha \dot{\alpha} \sigma \varepsilon \varsigma$, état clinique de toute une région à une époque donnée), on trouve qu'Hippocrate a reconnu de très nombreuses maladies: le causus, la phrénitis, la phtisie, l'angine, l'érysipèle, les aphtes, les tumeurs, les ophthalmies, l'anthrax, l'anorexie, l'hydropisie, la péripneumonie, la pleurésie... mais il faut constater que ces maladies ne sont pour lui que des faisceaux de symptômes, et qu'il ne les identifie pas vraiment dans leur spécificité. Ainsi, quelle est la différence entre un causus et une phrénitis? Dans Epid. III, douzième malade (III, 62-66 Littré), les signes suivants du causus sont indiqués: fièvre intense, soif, nausée, langue sèche, diarrhée, insomnie, hallucinations, vomissements bilieux; une description semblable se trouve au chapitre 6 (p.82). La phrénitis a l'air d'être une forme plus grave du causus, $d$ 'ordinaire accompagnée de transports et de délire (les phrènes sont considérés, c'est-à-dire le diaphragme, et c'est une localisation archaïque de la pensée). Que recouvrent ces deux noms de maladies? Des typhus, des rickettsioses, des paludismes, des attaques d'apoplexie, des maladies mentales? La confusion est totale. On comprend pourquoi quand on lit ce que dit Hippocrate dans Epid. III, 17, quatrième malade (III, 118, 5L.): malade atteint de phrénitis, les sueurs et les convulsions causèrent la mort. Le médecin ne voit pas que les sueurs et les convulsions sont des effets de la maladie, il les confond avec la cause, il met tout sur le même plan. De même, à côté de maladies nettement reconnues, il cite les maux de gorge, l'altération de la voix, les insomnies, les indigestions, la soif... Les symptômes et les diagnostics sont mélangés. Il y a donc une tentative pour trouver les causes des maladies et pour fonder une vétable nosologie, mais cette tentative n'aboutit pas, faute d'une anatomie et d'une physiologie suffisamment avancées. Le «point de vue synthétique» du pronostic hippocratique n'est pas un avantage, un idéal que les médecins devraient rechercher même à notre époque, c'est un défaut, un pis-aller, tout ce que pouvait élaborer une médecine qui était encore entièrement qualitative, qui ignorait la mesure (de la fièvre, par exemple), et n'avait pas de moyens d'investigation suffisants pour identifier les maladies, même si elle 
sentait que c'était nécessaire. Hippocrate a jeté les bases de la clinique, mais il n'en était encore qu'au tout début de cette évolution.

Naturellement, c'est dans les traités chirurgicaux et gynécologiques que le médecin hippocratique se rapproche le plus du diagnostic au sens moderne du mot, parce que là, la cause est apparente, au moins dans les meilleurs des cas. On a très souvent souligné (malgré le désordre dans lequel nous sont parvenus les traités, et quelques ignorances anatomiques peu explicables) l'admirable description de la chirurgie des membres qui se trouve dans Fractures et Articulations, ainsi que dans le traité des Blessures de tête. Il s'agit par exemple, dans ce dernier ouvrage, de distinguer si l'on a affaire à une contusion, une fracture ou un enfoncement crânien, et c'est dans ce texte (chapitre 10, III 214, 2L.) qu'on trouve le seul cas, dans la Collection

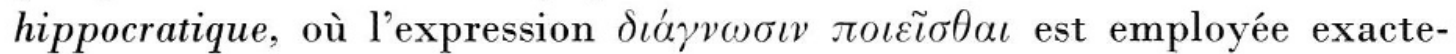
ment dans le sens moderne de «faire un diagnostic»: «Trouve-t-on l'os dénudé de la chair et lésé par le coup, on diagnostiquera d'abord l'état de l'os en examinant quelle est la grandeur du mal et quelle opération il exige. ${ }^{9}$

En gynécologie, le médecin ancien est gêné par le fait qu'à cette époque on n'osait pas examiner les femmes, on ne faisait que les interroger, et on employait des sages-femmes dans les accouchements. Des trois moyens d'investigation dont dispose le médecin ancien, l'interrogation du malade, l'examen, la palpation (et il y a des tentatives d'auscultation dans le troisième livre des Maladies), le gynécologue ne pouvait donc fonder son diagnostic que sur l'interrogation. Néanmoins on voit (Maladies des femmes, $I, 62 ; I I, 133,138)$ que le médecin commence toujours par des purgations et des saignées, c'est-à-dire qu'il suppose d'abord que la maladie a une cause humorale qui intéresse le corps tout entier, puis, si ce premier traitement n'a pas d'effet, il suppose qu'une partie a été lésée, et il intervient par des moyens mécaniques (bâtonnets, suppositoires, fumigations). Il n'y a donc pas lieu, une fois de plus, d'opposer à ce sujet le point de vue général au point de vue particulier.

Dans les maladies internes, le traité de l'Art, c. 11 (VI, 20 L.) reconnaît que le diagnostic est plus difficile que dans les maladies externes, mais «ce qui échappe à la vue du corps est saisi par la vue de l'esprit». On le voit dans les autres traités, le raisonnement doit logiquement suivre l'examen du malade. Et au besoin, ajoute notre auteur, si la nature se refuse à lui fournir des signes, le médecin peut les provoquer, en faisant prendre au patient des vomitifs ou des purgatifs, en lui faisant faire des exercices pour juger de sa respiration et 
de sa sueur. Nous avons donc ici un remarquable exemple de diagnostic provoqué.

En somme, on pourrait dire sans exagération que le diagnostic existe depuis le début de la médecine, mais qu'il n'a été vraiment mis au point qu'au $\mathrm{XIX}^{\circ}$ siècle. La médecine laïque est née à Babylone dès 1700 , et peut-être bien avant à Sumer, dès la fin du III $^{\circ}$ millénaire. De même en Egypte, les grands papyrus médicaux datent de 1550 (Papyrus Edwin Smith) et de 1250 (Papyrus Ebers) avant J.C., mais ils transmettent des connaissances qui sont certainement plus anciennes, qui remontent même peut-être jusqu'à l'époque des grandes pyramides. Or, il se trouve que la schéma d'une tablette akkadienne ou celui d'un papyrus égyptien est toujours le même: symptômes, diagnostic, thérapeutique, pronostic. C'est déjà le schéma de description des maladies qu'on trouve dans la Collection hippocratique, et que certains ont appelé «cnidien», mais il ne saurait être attribué à la seule «école de Cnide» ni à la seule médecine grecque. A vrai dire, c'est le schéma logique, il ne saurait y en avoir d'autre, puisqu'il reproduit tout simplement l'ordre chronologique de l'acte médical. Ce schéma a-t-il été inventé en Egypte ou en Mésopotamie? C'est difficile à dire, mais ce qui est certain, c'est que les Grecs l'ont emprunté des civilisations du Proche-Orient, comme une bonne partie de leur médecine, comme une bonne partie de leur civilisation. Or, ce schéma nomme la maladie, il comporte un diagnostic. Sans doute, à cette époque, ce diagnostic ne pouvait-il pas être plus précis que celui d'Hippocrate, il nommait les maladies seulement par une convergence de symptômes et certaines localisations, et les historiens de la médecine ont beaucoup de mal aujourd'hui à se mettre d'accord sur les maladies dont parlent les médecins babyloniens ou égyptiens, même avec l'aide de l'étude des momies. Le pronostic hippocratique englobe le diagnostic, il ne lui est pas systématiquement hostile. Dans les trois moments qu'il prétend réunir, rappeler le passé de la maladie, dire son présent, prédire son avenir, c'est le deuxième moment qui correspond à notre moderne diagnostic ${ }^{10}$. C'était là le moment essentiel de la médecine, mais il a fallu le travail de vingt-trois siècles pour en prendre conscience, et ce n'est pas la théorie du pronostic comme «vision synthétique» qui a pu faire avancer les choses, au contraire. Cette théorie n'était qu'une théorie empirique, comme les nosologies arbitraires qu'imaginaient à cette époque d'autres médecins, à Cnide ou ailleurs. L'histoire de la médecine nous enseigne, entre autres, que la science ne progresse jamais par des théories, des systèmes, mais toujours par le retour aux faits, et par l'élaboration de théories qui émanent des faits eux-mêmes. C'est sans doute 
ce qui s'est produit pour Hippocrate: il n'a inventé ni la théorie des humeurs, ni celle des crises et des jours critiques, mais il a dû remporter certains succès grâce à sa méthode d'observation clinique. Ensuite, il s'est passé ce qui se passe toujours: l'observation vivante est devenue dogmatisme, elle s'est heurtée à d'autres dogmes et a dû être remplacée par d'autres observations. Quant à l'hippocratisme moderne, qui prétend revenir à l'art du pronostic en négligeant les causes des maladies, refuser les antibiotiques et soigner par les plantes, ce n'est rien d'autre qu'une réaction contre la médecine expérimentale, qui n'a rien à voir avec l'Hippocrate historique. C'est de cette médecine prétendûment «naturelle» qu'il a fallu sortir pour inventer enfin une méthode permettant de soigner les gens et de sauver des vies.

\section{Notes}

1 Nous nous sommes surtout servi des Histoires de la médecine de A. Castiglioni, trad. J. Bertrand et F.Gidon, Paris 1931; C. Lichtenthaeler, trad. de l'allemand par Denise Meunier, Arthème Fayard 1978; M. Bariéty et C. Coury, Les grandes études historiques, A. Fayard 1963.

${ }^{2}$ Que tous les médecins de la Collection hippocratique aient admis l'importance des saisons, de la coction, de la crise et des jours critiques, c'est ce que nous nous sommes efforcé de montrer dans notre ouvrage Cnide et Cos? Essai sur les doctrines médicales dans la Collection hippocratique (Les Belles Lettres, 1981). Il nous a semblé qu'il fallait renoncer à l'opposition traditionnelle entre les écoles de Cnide et de Cos, et expliquer l'évolution de la médecine au $V^{o}$ siècle en Grèce par la substitution d'une physique d'origine éléatique (unité et invariabilité des éléments) à une physique d'origine ionienne (mutations et opposition des contraires).

${ }^{3}$ D'où l'expression «le triangle hippocratique», dont Madame Danielle Gourevitch s'est servie pour le titre de son ouvrage: Le triangle hippocratique dans le monde gréco-romain, Ecole française de Rome, 1984, qui montre autant d'esprit critique que de pénétration, et permet de rétablir sur bien des points la vérité sur la médecine anciennne.

${ }^{4}$ I. M. Lonie: Cos versus Cnidos and the Historians, Part I, in History of Science XVI (1978), Bucks-England, pp.42-75.

${ }^{5}$ L'amorce de la «question cnidienne» se trouve dans la nosologie que Galien attribue aux médecins cnidiens, et qui se trouve dans Affections internes, mais nous ignorons la source de Galien, et il est fort possible que cette nosologie soit tirée du traité lui-même; d'autre part, Galien cite, en l'attribuant à Euryphon, un passage des Sentences cnidiennes qui a de grandes ressemblances avec Maladies $I I, 68$. Cette indication est intéressante, mais ne prouve pas l'existence d'une «école de Cnide». Comme le dit W.D.Smith (Galen on Coans versus Cnidians, Bulletin of History of Medicine, XLVII, Baltimore, 1973, pp. 569-585), la théorie cnidienne est «auto-justificative»: elle tire ses preuves d'elle-même, c'est-à-dire qu'elle n'est pas prouvée. 
${ }^{6}$ Il est probable que, dès l'antiquité, les empiristes et les dogmatistes ont invoqué Hippocrate dans leurs discussions, et on imaginé des «Cnidiens» en face de lui. Galien a eu connaissance de ces débats, et les modernes ont ajouté leurs propres confusions à celles des anciens.

${ }^{7}$ Ceux qui soutiennent encore l'existence de deux écoles opposées tirent argument de la dernière phrase du Pronostic, qui dit: «Il ne faut pas demander les noms des maladies, car toutes celles qui se jugent dans les intervalles de temps indiqués sont décrites dans ce livre, car elles ont toutes les mêmes signes.» Cela veut-il dire que l'auteur du Pronostic est hostile à toute identification des maladies? Certainement pas, puisqu'il nomme et décrit plusieurs maladies dans son livre (tumeur à l'hypochondre, hydropisie, empyème, péripneumonie, céphalalgie, otite, angine...) et, comme le dit le commentateur tardif Stéphane le Sophiste (in F. R. Dietz, Scholia in Hippocratem, I, 231-32, Regimonti Prussorum 1834), Hippocrate n'a pas cherché à nommer toutes les maladies mais à indiquer tous les signes, et il en a fait son livre. Autrement dit, il a écrit un traité de sémiologie, tandis que les livres Maladies $I I, I I I$, Affections internes, sont des traités de pathologie. Il ne faut pas faire d'un procédé de composition une oppositon doctrinale.

8 Ancienne médecine, 19; Airs Eaux Lieux, 4, 6, 8, 9, 12, 15, 16, 20; Epid. II, 4, 5 («Il faut aller à la cause, et à l'origine de la cause»); Nature de l'homme, 13 («Quand les causes sont bien connues, il est facile de pronostiquer»); Affections, 25; Lieux dans l'homme, 31 («Il faut soigner les maladies à leur origine, mettre fin à l'origine de la maladie, et traiter»); Maladies des femmes I, 17, 62, II 133; Nature des os, 12, 13.

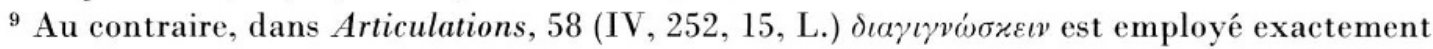
au sens moderne de "pronostic», tandis que les pronostics, les prévisions, sont appelés

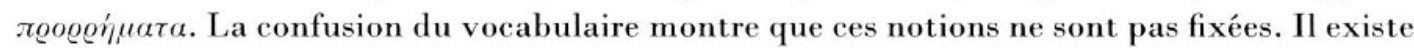
un seul texte dans la Collection hippocratique où diagnostic et pronostic ont l'air de s'opposer comme à l'époque actuelle, c'est dans le traité du Régime, $I I I, 69$ (VI, 606 L.), où il est dit

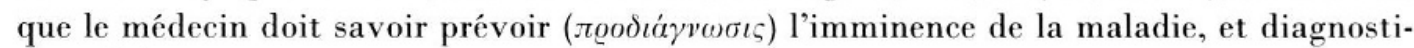
quer $(\delta \iota \dot{a} \gamma v \omega \sigma \iota \varsigma)$ si les aliments surmontent les exercices, ou le contraire. Mais il ne s'agit pas vraiment de diagnostiquer une maladie: l'auteur est persuadé qu'il a trouvé le moyen d'empêcher les hommes de tomber malades, en désignant exactement le point où l'équilibre entre les aliments et les exercices est rompu, dans un sens ou dans l'autre.

${ }^{10}$ Galien dit qu'Erasistrate (VIII, $14 \mathrm{Kühn}$ ) distinguait dans le pronostic hippocratique

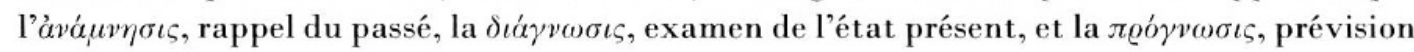
de l'avenir. S'il en est ainsi, le grand médecin d'Alexandrie pourrait bien avoir fixé notre vocabulaire dès le $\mathrm{III}^{\circ}$ siècle avant J.C. Cela est normal, si l'on songe que les Alexandrins ont fait faire de grands progrès à la médecine, grâce au développement de l'anatomie et de la physiologie, rendu possible par l'autorisation des dissections, donnée par les rois Ptolémée. 


\title{
Summary
}

Diagnosis and Prognosis in the time of Hippocrates and in ours

Nowadays, prognosis and diagnosis are clearly distinguished, but in the past it was not the same. In fact, diagnosis recently broke away from prognosis, with which it was confounded since antiquity. That happened thanks to the development of pathology, wich was made possible by anatomy and physiology during the classic age. In the time of Hippocrates, diagnosis was included in prognosis, and the physicians had no precise idea of specification of diseases. There is no doubt they were searching for a real diagnosis, like their predecessors in Babylonia or in Egypt, but they had no way to reach it. So the hippocratic prognosis is a makeshift, an empirical theory, as well as the various classifications of diseases, which took place in this time. Modern hippocratism is a mere reaction against experimental medicine, and has no historical foundation.

\author{
Antoine Thivel \\ Les Mimosas \\ Les Jardins de Cirniez \\ 26, Avenue de Flirey \\ F-06000 Nice
}

